Les frais annuels, y compris l'amortissement, seraient donc, suivant les cas, de 150.800 ou $167.000 \mathrm{fr}$. pour une production de $5.000 \mathrm{mc}$, ce qui correspond, par mc. de mélange de $7_{2} \%$ d'oxygène, à 3 c. et 3 c. 34 .

Ces chiffres sont très élevés, mais scraient notablement réduits pour des installations plus importantes.

Les fráis annuels ci-dessus indiqués correspondent à une production supplémentaire d'acide azotique de:

$780-600=180 \times 10.000 \mathrm{k}=\mathrm{r} .800 .000 \mathrm{kgs}$.

La masse d'air y contenue est de :

$$
x .800 .000 \times 0,22=400.000 \mathrm{kgs} \text {. }
$$

L.es prix de revient du kilogramme d'azote fixć, en supplément sont donc de :

$$
\frac{\mathrm{r} 55.800}{400.000}=0 \text { fr. } 39 \quad \frac{\mathrm{r} 67.000}{100.000}=0 \text { fr. } 42
$$

Dans ces conditions, le kilogramme d'azole fixé reviendra donc à $F_{s}: \frac{1,60+0,30 \times 0,39}{1,3}=1,32$ au licu de $\mathrm{I}$ fr. 6o sans la suroxygénation.

ou à Fs: $\quad \frac{290+0,30 \times 042}{1,3}=2,33$

au lieu de 2 fr. go sans la suroxygénation.

Cette amélioration est donc insuffisante pour ramener le prix du kg. d'azote à un chiffre qui permette de lutter contre la concurrence du nitrate du Chili. Il est par suite indispensable que nos chimistes parviennent à modifier le four électrique Birkeland-Eyde afin d'en augmenter le rendement dans une proportion sensible.

Paul Lévy-Salyador, Ingénieur des Constructions civiles attaché au Ministère de l'Armement et des Fabrications de guerre.

\section{LA HOUILLE BLANCHE ET LES ENGRAIS}

La Revue Chimie et Industrie, organe de la "Société de Chimie industrielle ", donne dans son $N^{\circ} 3$, sur la fabrication synthéthique des engrais de substitution au nitrate de soude du Chili, les renseignements suivants :

Il y aura en France, fin $1918,200.000$ HP de houille blanche affectés aux fabrications du carbure de calcium, de la cyanamide, du nitrate de chaux et de l'acide azotique. Si cette puissance était uniquement employée à la fabrication de la cyanamide, elle en donnerait une production annuelle de roo.0oo tonnes.

En I9r3, on n'en produisait que 8.000 tonnes en France et 30.000 tonnes en Allemagne, qui porte cette année sa production à 400.000 tonnes $1 . .$.

D'autre part, le procédé HABER, mis au point cn Allemagne et qui y donnait 30.000 tonnes de sulfate d'ammoniaque en rgr3, produira 50o.0oo tonnes en rgr8.

En r9r3 notre Pays ne consommait que 300.000 tonnes de nitrate du Chili - ce qui représente 7 à 8 kilos à l'hectare, contre i5 kilos en Angleterre et 18 en Allemagne.

Avant la guerre, les "Empires Centraux " et les EtatsUnis absorbaient annuellement r.500.000 tonnes de nitrate de soude, l'Allemagne en prenant 800.000 à sa part.

\section{$\star^{\star} \star \star$}

Ces chiffres nous montrent ce que nous avons à faire. Après le nitrate de soude naturel, et à son défant, c'est le sulfate d'ammoniaque qui répond le mieux à l'emploi universel, de l'avis unanime des agriculteurs.
La fabrication de ce produit, lo plus apte des fixateurs d'azote par voie élcctrosynthétique, suivant la conception envisagće dans notre éludc sur " ILe rôle du Rhône aménagé dans les industries électro-métallurgiques et chimiques " ${ }^{1}$ ), est donc susceptible d'une ampleur plus grande encore.

Il faudrait alors démontrer à l'évidence le besoin des encouragements et des moyens de travail à donner à nos chimistes pour mettre au point la transformation de l'ammoniaque en aride azotique et subséquemment en nitrate de potasse, avec un prix de revient permettant sa substitution au nitrate du Chili dans les circonstances actuelles.

Il est question ici de potasse au lieu de soude et de chaux parce qu'il faut compter que la Victoire de nos armes nous remettra en possession des fameux gisements de Crrnay dont les Allemands tirent 5.000.000 de tonnes par an.

\section{E.F Corr.}

\section{LES CONSÉQUENCES DE L'AMÉNAGENENT DU RHONE POUR L'AVENIR INDUSTRIEL \\ DES BASSINS VERSANTS DE LA RIVE DROITE}

Le problène de la navigation du Rhòne, combiné avec l'utilisation de ses forces motrices et avec l'irrigation agricole, est à l'ordre du jour, il esl permis d'en escompter après la guerre la prompte solution. Chaque District intéressé, dans la vallée du Fleuve, doit donc se préparer à tirer parti, au fur ct à mesure de la réalisation de cet aménagement, des ressources nouvelles qu'il lui apportera.

Les études et les travaux que réclament ces utilisations locales ne sonit pas moins nécessaires que ceux qu'exigera le projet principal. Ne faut-1l pas, en effet, que les communes riveraines aient des ports bien disposés pour pouvoir recourir à la batellerie ? Et les cantons de l'intérieur, qui communiqueront avec les ports fluviaux par leurs chemins de fer transversaux, n'ont-ils pas besoin que les gares terminus soient reliées avec les quais, condition indispensable pour l'embarquement ou le débarquemènt de léurs marchandises ? Chaque localité de la rive drojte on de la rive gauche, vivaraise, languedocienne, dauphinoisc ou provençale, doit par conséquent dresser son programme particulier et se tenir prête à l'exécuter, afin de n'être pas en retard lorsque s'élaborera l'œuvre principale.

Comme je viens de l'indiquer, il y aura deux modes d'utilisation bien distinctis. Les communes littorales accèderont directement au Rhône par leurs ports respectifs. Lees diż tricts intéricurs emprunteront la voie ferrée sur r5, 20, 3a ou 40 kilomètres el au-delà avant de joindre te fleuve Mais n'existe-t-il pas, cll certaine- situations, un troisième cas qui, bien que plus rare, mérite d'être envisagé et inscrit sur la liste des travaux?

Le Rhône reçoit, par chacune de ses rives, plusieurs affluents entre Lyon et Arles Ces affluents et leurs tributaires sont déjì ou seront à brève échéance aménagéz en vue des forces hydro-électriques qu'ils peuvent donner. N'y aurait-il pas lieu, sinon pour tous, du moins pour certains, de combiner ces chules érholonnćes avec des canalisations à ćcluses accersibles à la batellerie, et prolongeanl la voie navigable du fleuve à une plus ón moins grande dislance dans l'intérieur des terres?

(1) La Houille Blanche: No 152. Mai-Jum 1918; et (Nov.-Dec. 1917) 